\title{
Image Compression Analysis in Torrent Based Wireless Sensor Network
}

\author{
${ }^{1}$ Bhushan N Mahajan, ${ }^{2}$ Dr Prof. Anjali. R. Mahajan, ${ }^{3}$ Prof A. Thomas \\ ${ }^{1}$ Research Scholar, G H Raisoni College of Engineering, Nagpur \\ ${ }^{2} \mathrm{HoD}$ (IT) Govt. Polytechnic College, Nagpur \\ ${ }^{3} \mathrm{HoD}$, (CSE), G H Raisoni College of Engineering, Nagpur \\ Email:bnmahajan78@gmail.com,armahajan@rediffmail.com,tinav2@gmail.com
}

\section{Received: 09 ${ }^{\text {th }}$ July 2018, Accepted: $14^{\text {th }}$ August 2018, Published: 31 ${ }^{\text {st }}$ August 2018}

\begin{abstract}
Image compression can be achieved efficiently using Wavelet transformation concept in wireless sensor network. Torrent based architecture can be plugged successfully in wireless sensor network. The results are analyzed in this study and outcome of experiment is stated with respect to performance parameters. Wavelet is a template to record changes those occur in real-world data or signals. These changes may occur abruptly, smoothly or frequently. In other words, Wavelet represents a slowly altering contrast or brightness. Images have some smooth and abrupt regions in one specific place and the scanning proceeds in any of the direction, these regions are interrupted by abrupt changes in color or brightness or contrast. It results in gathering the most interesting part of the image data. One can categorize the change in term of contrast or brightness data. One may record this change in terms of the waves, image metadata or coefficients array and thus achieve image compression. Torrent based architecture multiply the processing capabilities of a cluster of sensor nodes. The single node cannot handle heavy processing task, a large number of bytes and communication at a time. Hence processing schedule and communication schedule management algorithms are needed to be designed and allocated separately to a specific set of nodes.
\end{abstract}

Keywords: Wavelet Transformation, Image Compression, Wireless Sensor Network, Torrent Based Architecture, TDMA Based.

\section{Introduction}

In today's era, the use of digital images is increasing. The next issue is how to store the uncompressed digital images on media and how to transfer them from one machine or node to another node. The compression can save the space on media and bandwidth required for data. For web this issue is more crucial. In case of sensor network, this issue is more crucial. Hence high degree of compression is needed. There are verities of image formats. Ex- JPEG, GIF, tiff and so on. So various types of encoder and decoder are invented. Most common we know is DCT based Encoder and Decoder. Compressions are either lossless or lossy.
Modes may be either Hierarchical, progressive or sequential. Due to low bit rate, performance of Encoder and Decoder decrease. Image may be color or grey. Multiple greyscale images are combined to produce color image. Each input $8 \times 8$ pixel block is individually compressed. The output $8 \times 8$ blocks are merged to produce one compressed image.

Wavelet transformation is also another type of compression strategy. Wavelet shows approximation and it compare standard patterns of signals with the given input pattern of signal. It provides higher compression ratio. Based on wavelet, various schemes have emerged now a day. Wavelet can be scalable, shifted and thus whole image is transformed. Hence, more compression can be achieved. Progressive image transmission can be also achieved. If one want to data analysis, Fourier transform is the best tool. The limitation is the Fourier transform cannot give the broad changes that efficiently. The data is represented using Fourier transformation using addition of input sine waves. Base wave is multiplexed with carrier wave and the result waves are produced. Similarly, sine waves are summed, and resultant wave is produced.

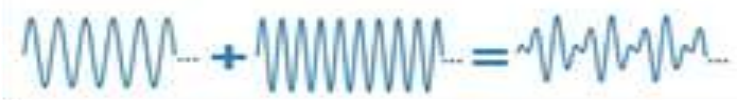

Fig 1: Sinusoidal Waves

Another way to look at wavelet is wave having fixed duration oscillation with zero mean. Wavelet does not exist for infinite duration. Wavelets can be thought of a collection of sample thumbnail snap of waves or templates. They come in different sizes and shapes. One has to match it with the input signal to be compressed.

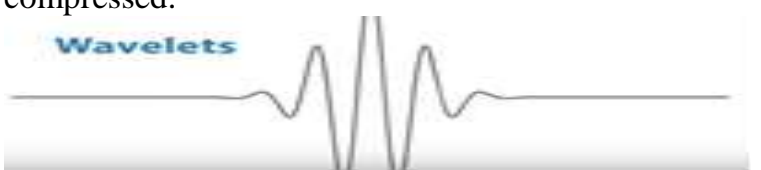

Fig 2: Sample Wavelet

The next step is to choose the most appropriate wavelet for the given image to be compressed. The wavelets are scaled and shifted to fit the curved regions or changes of input signal. 


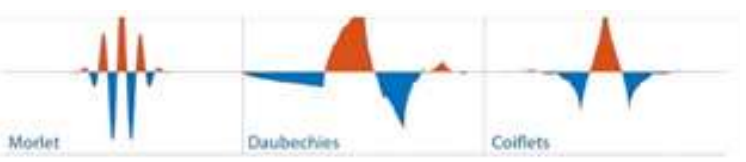

Fig 3: Types of Wavelets

\section{Scaling}

Scaling of signal means extending signal in time domain. We can consider it as extending or stretching signal horizontally. Mathematically, stretching signal is represented as given below. Here $\mathrm{S}$ is called as scaling factor which is a positive value inversely proportional to frequency. The impact is, if the signal is stretched more, the frequency of signal will be decreased. Thus, reciprocal relation exists between scale and frequency with the constant of proportionality. The constant of proportionality is called as central frequency.

$$
\Psi\left(\frac{\mathrm{t}}{\mathrm{s}}\right) \mathrm{s}>0
$$

Fig 4: Stretching Equation of Wavelets

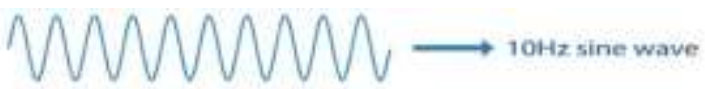

Scaling the $10 \mathrm{~Hz}$ sine wave by 2

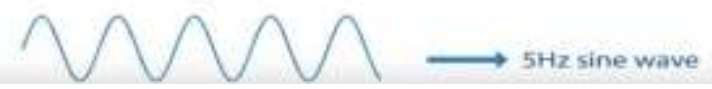

Fig 5: Scaling of Wavelet

In frequency domain, these wavelet shows bandpass characteristic. Below given equation shows Equivalent frequency where $\mathrm{Cf}$ is central frequency as explained above and sampling interval is delta t. Below given table represent how change in wavelet scale impact on Equivalent frequency. If you use larger scale value or larger $\mathrm{s}$ value, it will result in a larger stretched wavelet. If you use smaller scale value or smaller s value, it will result in a smaller stretched wavelet.

$$
F_{e q}=\frac{C_{f}}{s \delta t}
$$

\begin{tabular}{|c|c|c|c|c|}
\hline $\begin{array}{l}\text { WAVELET } \\
\text { SCALE }\end{array}$ & $z$ & 4 & B & 16 \\
\hline $\begin{array}{l}\text { EQUIVALENT } \\
\text { FREQ (F.al) }\end{array}$ & $\frac{F_{t u}}{z}$ & $\frac{r_{-1}}{4}$ & $\frac{f_{m-1}}{B}$ & $\frac{5}{10}$ \\
\hline
\end{tabular}

\section{Fig 6: Scaling Equation}

Fig 7: Scaling Wavelet by a Factor

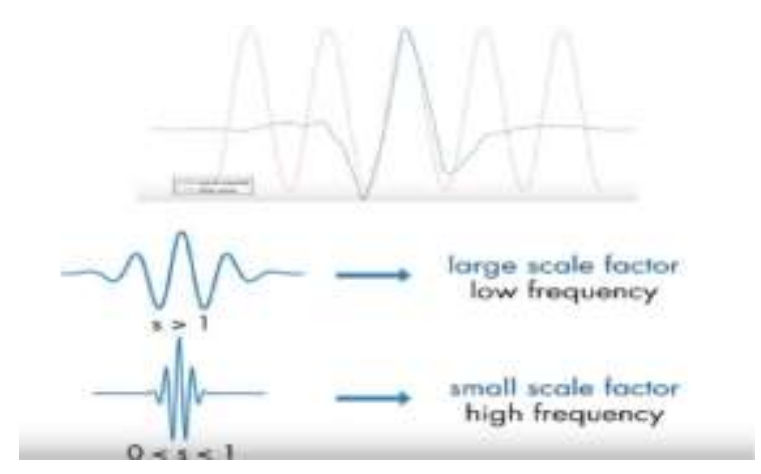

Fig 8: Scaling Factors

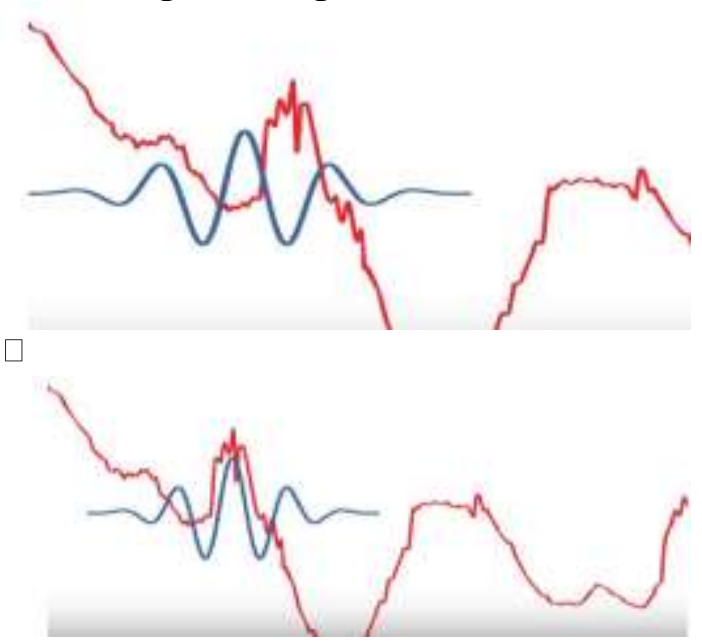

Fig 9: Matching Wavelet Templates

Above given is a target wave. To record changes in different part of the wave, we need a template wavelet photo. Let us match the template wavelet photo with the various curved regions of above wave. It can be thought as you are doing a tracing on drawing worksheet and overlapping the matching pieces of the curves or standard template curved blocks over the various regions. If you get a match, you record the curve id. If you need to do stretching, you do stretch the curved block till it matches the target region.

Sine wave of same frequency a large scale factor results in a stretched wavelet which corresponds to lower frequency a smaller scale factor results in a snack and wavelet which corresponds to high frequency wavelength helps in capturing the slowly varying changes in a signal while compressed Thus, conclusion is wavelet helps in capturing the abrupt changes when you start scanning various regions of wave and you can construct different scales that inversely correspond to the equivalent frequencies .

\section{Shifting}

In some cases, the wavelets are shifted horizontally or vertically to match the curved regions of signal. Shifting is also known as delaying signals. Wavelet is said to be shifted and centred at $\mathrm{K}$. 


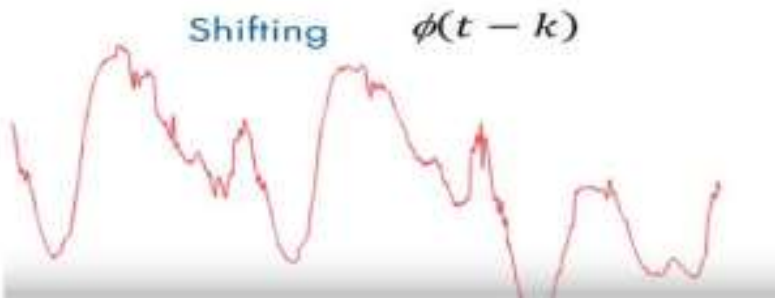

Fig 10: Wavelet shifting Equation

Wavelet is shifted vertically up or down. Thus, it is aligned with the curved region. The wavelet is scaled and shifted. Thus, continuous and discrete transformation can be achieved. There are various applications of continuous and discrete transformation of wavelet. These are used for removing noise from the signal. These are used for compression of signals. Based on various values of translation parameters and scaling factors we can achieve different results.

\section{Continuous Wavelet Transform}

In this case, we obtain time frequency analysis of signal. For time frequency analysis, Analytics wavelet are best suited. Analytics wavelet are suitable because these wavelets do not possess negative frequency component.

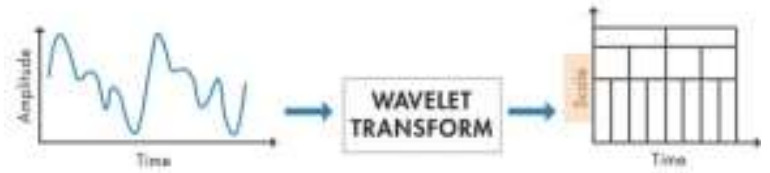

Fig 11: Continuous Wavelet Transform

Scaler wave is taken. It is scaled by a factor of 2 . This result in reduction in equivalent frequency by a factor of 2. Within ach octave, we can analyse the signal. Thus, we receive number of scales for analysis. If one receives more number of scales, finer will be the discretisation. These scales are multiplied by sampling interval of signal. Below given is the scale for Bump wavelet. Here, 32 octave scale is used.

\section{Wavelet Scales}

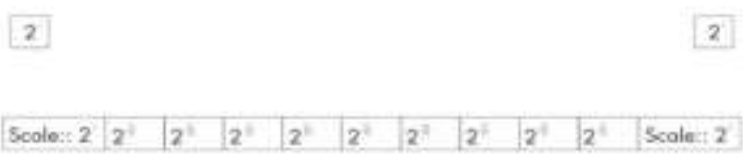

Fig 13: Wavelet Sampling

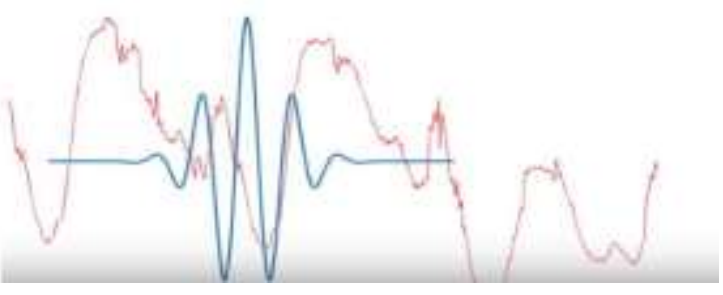

Fig 14: Wavelet Match in CWT

One can alter this procedure for every one of the scales bringing about Coefficient that are the capacity of wavelet scale and move parameter. To place it in context the signal with 1000 examples and broke down with 20 scales brings about 20000 coefficients. Thusly, you can better portray oscillatory conduct in signals with the ceaseless wavelet change.

\section{Discrete Wavelet Transform}

This wave transformation is very useful for denoising. This is also useful for compression of signals. They represent naturally occurring signals. These involve coefficients. First, base scale need to be set. If we raise base scale, different scales are obtained. It is called as dyadic scaling and dyadic shifting. This transformation reduces number of redundant coefficients. One obtains same number of coefficients as that of length of input signal. It requires less coefficients and hence it require less storage. We can simulate it as multi rate filter bank. We can simulate a signal $\mathrm{s}$ is there. It is passed through High pass filter and low pass filter. It results in High pass and low pass sub bands. Half of the sample signals are discarded in this process.

The number of coefficients are small in this case. Sub bands can be reconstructed by cancelling aliasing if occur due to down sampling. By using iterative low pass filtering, narrower and narrower sub bands are achieved.

$$
\begin{aligned}
& \text { Scaling } \\
& 2^{i} \quad(j=1,2,3,4 \ldots) \\
& \text { Translation } \\
& 2^{i n} \quad(17=1,2,3,4 \ldots)
\end{aligned}
$$

Fig 15: Discrete Wavelet Transform

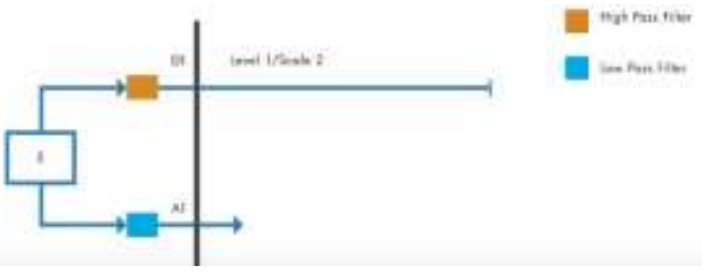

Fig 16: High Pass and Low Pass Filtering 


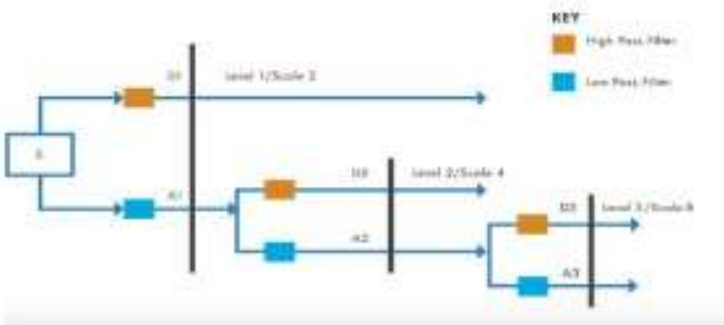

Fig 17: Iterative Filtering

\section{Lifting Scheme Algorithm}

Data is splitted into odd and even sets. Then predict odd set from even set. It ensures polynomial cancellation in high pass. Then update even set by using wavelet coefficients to calculate scaling function. It ensures prevention of moments in low pass. It requires half number of computations. Implementation is faster. It is suitable for low power application. No auxiliary memory needed. It ensure loss less compression. It can be used for irregular sampling.

\section{Haar Wavelet}

Alfred Haar introduced it. Two types of coefficients are obtained from it. Course approximation of speech and Fine details of speech. It involve forward and Inverse transformation. In forward transformation, scaling coefficients are calculated. Then wavelet coefficients are calculated. In Inverse transformation, simply addition and subtraction is required. Average a $=(\mathrm{x}+\mathrm{y}) / 2$ and Difference $\mathrm{d}=(\mathrm{x}-\mathrm{y}) / 2 \cdot \mathrm{m}=(\mathrm{a}-\mathrm{d}) / 2$ and $\mathrm{n}=(\mathrm{a}+\mathrm{d}) / 2$. First row by row transformation is done. Then column by column transformation is done. $8 \times 8$ Matrix used is given below.

$\left[\begin{array}{cccccccc}1 & 1 & 1 & 1 & 1 & 1 & 1 & 1 \\ 1 & 1 & 1 & 1 & -1 & -1 & -1 & -1 \\ \sqrt{2} & \sqrt{2} & -\sqrt{2} & -\sqrt{2} & 0 & 0 & 0 & 0 \\ 0 & 0 & 0 & 0 & \sqrt{2} & \sqrt{2} & -\sqrt{2} & -\sqrt{2} \\ 2 & -2 & 0 & 0 & 0 & 0 & 0 & 0 \\ 0 & 0 & 2 & -2 & 0 & 0 & 0 & 0 \\ 0 & 0 & 0 & 0 & 2 & -2 & 0 & 0 \\ 0 & 0 & 0 & 0 & 0 & 0 & 2 & -2\end{array}\right]$

Fig 18: $8 * 8$ matrix

\section{Materials and Methods}

We will try to understand the basic characteristic, the basic nature of the wireless network. How the wireless network is replacing the communication pattern will be needed to understand. We have a fixed wireless network where we could have a microwave as a backbone and it is very communicative. Wireless device communicates and then send the data to a satellite-based backbone network that helps you to communicate from here to a particular space station and then it communicates with satellite [1]. That means there are stations which are interconnected through Microwave and they are fixed at the place and they do not change their position. Fixed Area Network and second is satellite backbone. When we launch satellites, they attain geosynchronous orbit that is $36000 \mathrm{~km}$ from the earth. If a satellite is launched, then it rotates at the same speed as the Earth rotates. Hence, the satellite will be covering the same position all the time, but one satellite is not sufficient to cover the entire group, so we need multiple minimum three or four satellites. It means we are grouping satellite and forming a cluster.

While making calls to our friends and colleagues if you do it using satellite communication, the distance between a satellite and geosynchronous is $36000 \mathrm{~km}$ and for the signal to go from Earth to the satellite it takes 207 milliseconds in communication. 270millisecond time is a good time [2]. If you send a signal to a satellite and geosynchronous orbit and getting a response will take 540 millisecond that is to you that time as far as wireless networks.

Therefore, we should know Orbit satellite will take the time to send a signal as well as to get a response. The speed and the relative velocity of the satellites will be very high relative to the earth that means they may be not focusing all the time at the same position. They will be changing the position in different areas on earth at the time, so it makes more difficult to communicate [3]. The channel which the system user is wireless. That means there is no wide medium and all communication is taking place through the wireless. The network access the services and beyond that your mobile phone access the service.

\section{Cellular Network}

Base station creates a cell and any mobile handset falling within that radius of a base station will only communicate with that base station. Hence, we call it as a cellular network. The cellular network work with mobile only through signals from your mobile handset to the base station. [4] Internet connection from your home modem to the service provider is through a modem. You are accessing the services provided by service provider. The service access is provided through a wireless medium and that too through your modem. In India, you have you call a broadband connection provider. The speed provided is 256 or 512-kilobytes of data transmission. In some case, broadband speed is considered as more than 2 Mbps. In short, there is no any fixed definition. [5]

\section{Sensor Network}

If you have a sensor network, you are allowed for collecting information and transmitting this information to a central Node. If the need arises you want to communicate this information to the rest of the word, then you need connectivity with the internet. Gateway provides you connectivity to the internet. If you look at the composition of a sensor node how is sensor node consists of and what are the various components of a sensor, it does have few components 
which do the task of sensing whatever activities or event or a phenomenal it is supposed to send, and it is supposed to measure. For example, temperature, for this sensor component will be required and it will measure the temperature. The node has to process that information, so node should have a dedicated processing Unit and algorithm or code where it can process the data using the algorithm.[6] Data is sent by the sensor and then what you need is sensor node must react to incoming commands. Sensor nodes should have a component which provides you with the capability for wireless transmitter and receiver. That component will transmit and receive data. When node collects data the phenomenon which is being measured by the sensor, the data must be stored in some cache. The sensor node must have some storage device or some memory support. Sensor nodes need a power supply.[7] We deploy the sensor nodes at various places with may be accessible or sometimes very difficult to access by the human beings. Therefore, what you need is there must be provided with the DC power supply. That power supply cannot be AC power supply for sensor nodes. These batteries have limited power supply and when they are used, the power will drain and gradually this power may so drain that the battery is dead. [8] Now you need to recharge this battery. But sensor node if is an accessible area where you can go and replace the battery this is done. But if the senses are deployed at a place where the human accessibility is not possible then the only way out is this sensor must be replaced by new one. Therefore, the cost of sensor involved in some applications must be cheap. But at the same time for human safety, they may be very useful. For monitoring the animal's movement, the animal will have a sensor node and one will put it on the animal body and that will send a signal. The sensor will be containing a GPS receiver which may be communicating with the satellite GPS satellite and gathering information. Using the location information, the latitude and latitude of that place where the animal has moved will be made available.[9] These are called GPS module. Along with the GPS receiver, we can put on the air temperature sensor, the humidity sensor and transmission power must be sufficient to transmit it to the receiver depending on the distance.

\section{Design}

In the Wireless sensor network, the torrent seed-based system is the proposed for image data sharing system, maintains a collection of central servers to facilitate interaction like file exchange between distributed seeds. The central servers give content classification and search services, whereas the content itself is changed amongst the seeds directly through the central servers to make it able to search content expeditiously and accurately. The reason for failure may be due to failure at the server. For this reason, the torrent systems assume a partly or a strictly redistributed design. The same system can be incorporated in the wireless system.[10] In partly redistributed systems or hybrid torrent seed-based systems, there exists a hierarchy of sensor leaf nodes and distributor seed Nodes. The aim is to fulfil the necessity of quantification and search potency. Also, the processing power is added and multiplied by a large extent due to the presence of various nodes. The role of every seed node is appointed dynamically and altered as per the need-based the on its remaining capability and up to date activity.

The seed Nodes are inter-connected, forming an additional compact higher layer network for the sake of search potency. There's no logical correlation between nodes and content at intervals the network, thus locating content should use a brute force approach. Specifically, once a seed Node receives a question from one node, it initial checks its native content index. If the question cannot be solved, the seed Node forwards the question to different connected seed Nodes.[11] This is in distinction to the normal client-server models for multimedia system communications that use dedicated networking infrastructure. Applications of the torrent seed-based multimedia system are torrent seed-based system embrace non-centralized file sharing and rising content distribution and multimedia system sensing element networks.

The paradigm shift to less stratified and additional localized multimedia system networking needs that several basic broadband communication challenges be revisited.

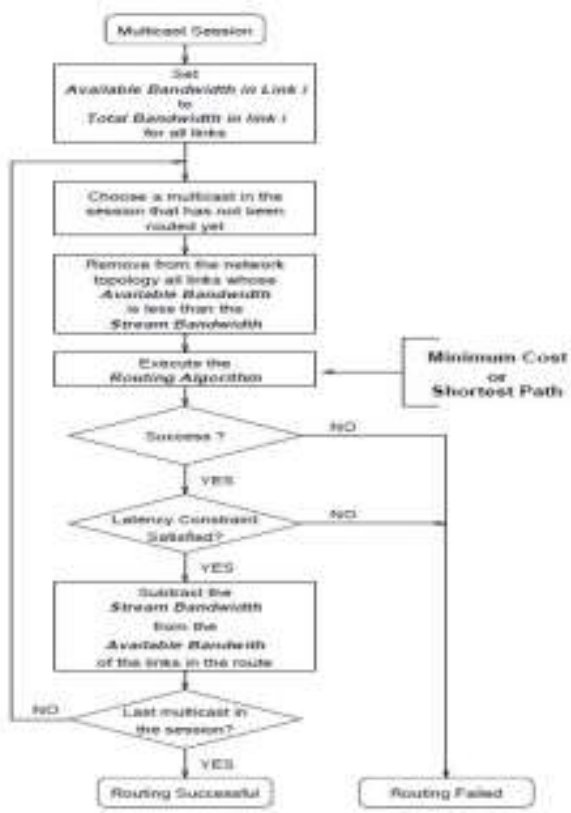

Fig 19: Routing Decision 


\section{Algorithm \\ Algorithm for cooperative streaming of bytes in a torrent seed-based system using TDMA approach Step 1 -}

At Seed node $\mathrm{S} 1$.

Take Clock reading (Say r1) and refer Packet arrival chart.

Find out queue identifier (Say q1)

Find its incoming time (r2)

Find out the difference $(\operatorname{diff}=\mathrm{r} 1-\mathrm{r} 2)$

Initialize wakeup time $=(\mathrm{r} 1+$ diff $)-1$

Go to sleep until wake up time. (Sleeping saves

battery life) Wake up at wakeup time.

$\mathrm{S} 1$ will confirm from a neighbour, Does the packet arrived? If the Response code is Yes, give a token of identification and receive a packet from N1.

If Answer is No, then wait for next $\mathrm{n}$ second. Again, ask N1. Once Packet arrived, Neighbor $n 1$ will send an alert message to all seeds s1 to s9.

$\mathrm{S} 1$ will give the token to the neighbour node.

S1 will Receive packets \# 1 from a neighbour and start processing on it. Ex - decompression. Extract bytes. At Next seed S2,

Receive packets \# 2 from a neighbour and start processing on it. Ex - decompression, Extract bytes. Thus, parallel execution takes place.

\section{Step 2 -}

All seed nodes will start processing on their bytes chunks in parallel. When all node did processing, they all send signal of task completion.

Step 3 -

The bytes are now converted to the raw format and ready to play by the player.

We can modify the above-given algorithm. Option 1 is each node should have complete info on the network. Option 2 is each node should contain info of 1 hop nodes and if alternate node not found. It should share a common node with the neighboring node. They should create Triangle like structure in case of nonavailability of the alternate node. Option 3 is that some administrative group node or head node should provide correct info to all node. Nodes can decide and read that where the common or non-common routes is. Option 4 is Set of forwarding path node and Reverse path node is supplied by Head node. Other should actively test and follow it. It would be easy to reach up to Source as well as Destination.

Set a max limit of how many no of hops RREQ should take to reach up to Source. Set a max limit of delay RREQ should take to reach up to Source. Using a single path or using multiple paths, which is the best approach for data transmission?
1. First primary path is used. After primary path does not work, the secondary path should be used.

2. After secondary path exhausted, other alternate paths are used.

3. A number of routes, more routes maintenance are required.

4. Simultaneous path usage and their maintenance of selected path are required.

5. If a node fails, the route should select an alternate node (using hello packets) and maintain the integrity of path.

6. On multiple paths, we can flow data packets by splitting packets or by replicating packets.

7. Different addresses can be used for different paths.

8. Instead of waiting for node failure, we can monitor path and node health after six rounds of the test. It avoids rediscovery of routes.

Here, it is assumed that a grid-like structure of $10 \times 10$ nodes is created. Consider a node having surrounding pairs of 6 nodes in star-like fashion having the middle node as path selector. All 6 are in communication range of the central node. Other 6 surrounding nodes act as 6 alternate nodes. Central node, lower node and upper node are part of the primary path. Central node can divert traffic through the alternate nodes based amount of remaining energy. Using any of the 6 nodes, the secondary path can be created.

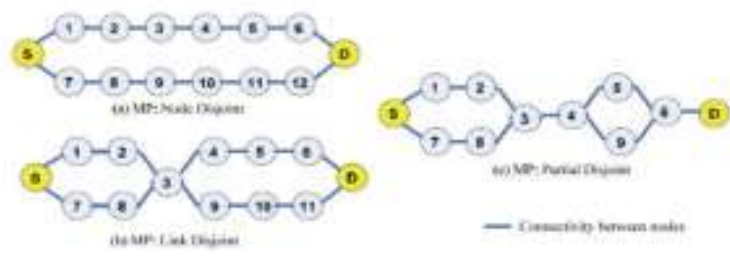

Fig 20: Common Link Between Clusters

Through node, three types of packets flow. Transmitted packet, received the packet and relayed packets. Those may be data packets or control packets. This information is used to determine path life for next round of transmission. Path selector node treats neighbour as a cache. Each path selector node keeps the no of neighbour available to it. Using neighbour, they create primary and secondary and alternate paths.
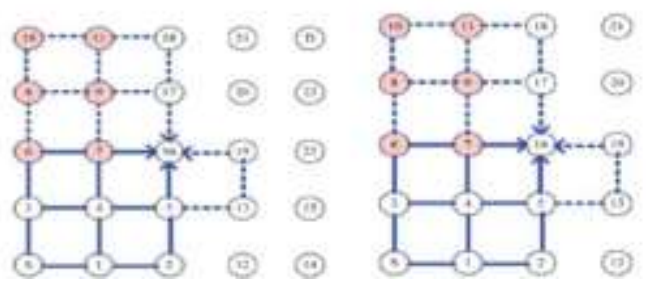

Fig 21: Cooperative Accumulation of Byte Content from All Seeds in Each Grid 
Each packet when transmitted or received, some amount of energy of a node is spent. It directly affects path life. The node should not interfere in working of any other node. Each neighbour node when work, some amount of cache it provides for the path. Path selector node act as a carrier of control packets or cache packets. Neighbour node act member of the primary path or secondary path

\section{Result and Discussion}

Below is the result with respect to the compression and transmission approach. Multimedia content distribution may be a distinguished application space of TORRENT SEED networks. As torrent seed metric linear unit technologies are still evolving, it opens various analysis issues.

\begin{tabular}{|l|l|l|}
\hline File Size & Transmission Delay & Compression Type \\
\hline $6 \mathrm{~KB}$ & $8.5 \mathrm{sec}$ & JPEG \\
\hline $5 \mathrm{~KB}$ & $6.3 \mathrm{sec}$ & HAAR \\
\hline $8 \mathrm{~KB}$ & $12.0 \mathrm{sec}$ & $\begin{array}{l}\text { Colour Index } \\
\text { Mapping }\end{array}$ \\
\hline
\end{tabular}

Table 1: Transmission Delay Chart

\section{Conclusion:}

Multimedia content distribution is of great demand nowadays. The content distribution can be achieved by emerging Torrent seed nodes networks. It includes:

Cost, peer organization and system potency in content access and distribution of media content. Reliability/robustness, system's adaptation capability to the dynamic network and different environmental conditions is mandatory. The image frame rate matters in this case.

Hence image compression should be done within a stipulated time. The optimized and best method of image compression are jpeg and HAAR wavelet. For transmission of data, nodemcu ESP32 or ESP 8266 can be the used.

\section{References}

[1] Song Ci, Dalei Wu, Yun Ye, Zhu Han, Guan-Ming Su, Haohong Wang, Hui Tang, "Video Summary Delivery Over Cooperative Wireless Networks". Ieee Wireless Communications. (April 2012)

[2]. Ivan Kastelan, Mihajlo Katona, Goran Miljkovic, Tomislav Maruna, Mirko Vucelja, "Cloud Enhanced Smart Home Technologies", Ieee International Conference On Consumer Electronics. ( 2012)

[3]. Lei Lei And Zhangdui Zhong, Chuang Lin, Xuemin (Sherman) Shen, "Operator Controlled Device-To-Device Communications In LTEAdvanced Networks", Wireless Communications. (April 2012)
[4]. Jun Zhang And Khaled B. Letaief, "Interference Management With Relay Cooperation In Two-Hop Interference Channels", Ieee Wireless Communications Letters, Vol. 1, No. 3. (June 2012)

[5]. Jin-Bum Hwang And Chae Y. Lee, Member, Ieee, "Effective Video Multicast Using Svc With Heterogeneous User Demands Over Tdma Based Wireless Mesh Networks", Ieee Transactions On Mobile Computing. (2012)

[6]. M. Majid Butt,"Energy-Performance Trade-Offs In Multiuser Scheduling: Large System Analysis", Ieee Wireless Communications Letters, Vol. 1, No. 3. (June 2012)

[7]. P. Ubaidulla, And Sonia A Issa," Senior Member, Ieee, "Optimal Relay Selection And Power Allocation For Cognitive Two-Way Relaying Networks", Ieee Wireless Communications Letters, Vol. 1, No. 3. (June 2012 )

[8]. Nof Abu Zainab, Anthony Ephremides, "Energy Efficiency Of Cooperative Relaying Over A Wireless Link", Ieee Transactions On Wireless Communications, Vol. 11, No. 6. (June 2012)

[9]. Kushal Dutta “A Smart Job Scheduling System For Cloud Computing Service Providers And Users: Modeling And Simulation", 1st Int'l Conf. On Recent Advances In Information Technology Rait, (2012)

[10].Özgü Alay, Student Member, Ieee, Pei Liu, Member, Ieee, Yao Wang, Fellow, Ieee, Elza Erkip, Fellow, Ieee, And Shivendra S. Panwar, Fellow, "Cooperative Layered Video Multicast Using Randomized Distributed Space-Time Codes", Ieee Ieee Transactions On Multimedia, Vol. 13, No. 5, (October 2011)

[11].Zhangyu Guant\$, Lei Ding \$, Tommaso Melodiał, And Dongfeng Yuan†, "On The Effect Of Cooperative Relaying On The Performance Of Video Streaming Applications In Cognitive Radio Networks", Ieee Icc Proceedings. , (2011) [12] R. \& Srivastava, M. Ganeriwal, S.; Kumar, "Time Sync Protocol For Sensor Network, The First Acm Conference On Embedded Networked Sensor System (Sensys)", Los Angeles, Pp. 138- 149, (Nov 2005) 